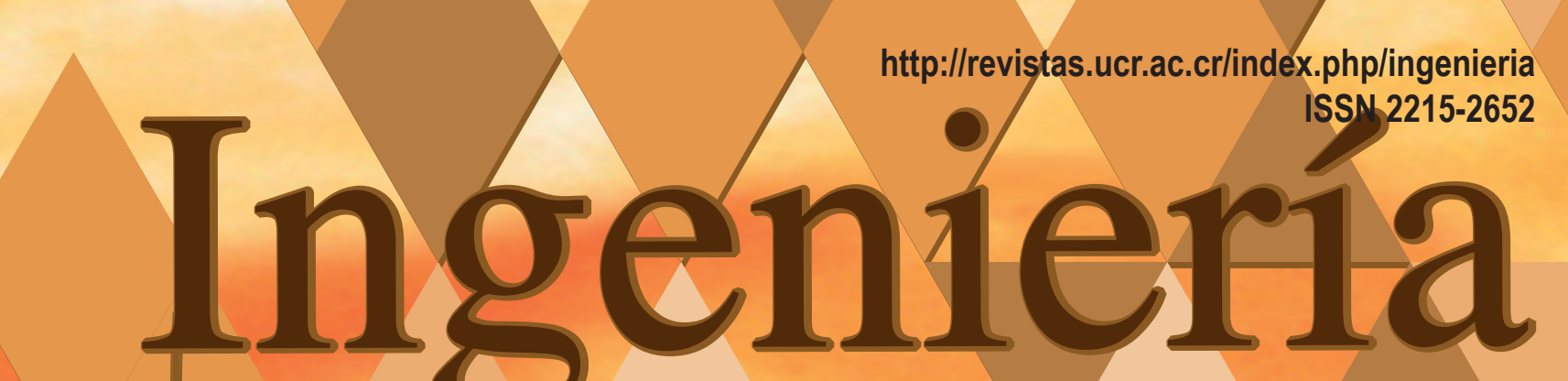

Revista de la Universidad de Costa Rica FEBRERO/AGOSTO 2014 -VOLUMEN 24 Número (1)

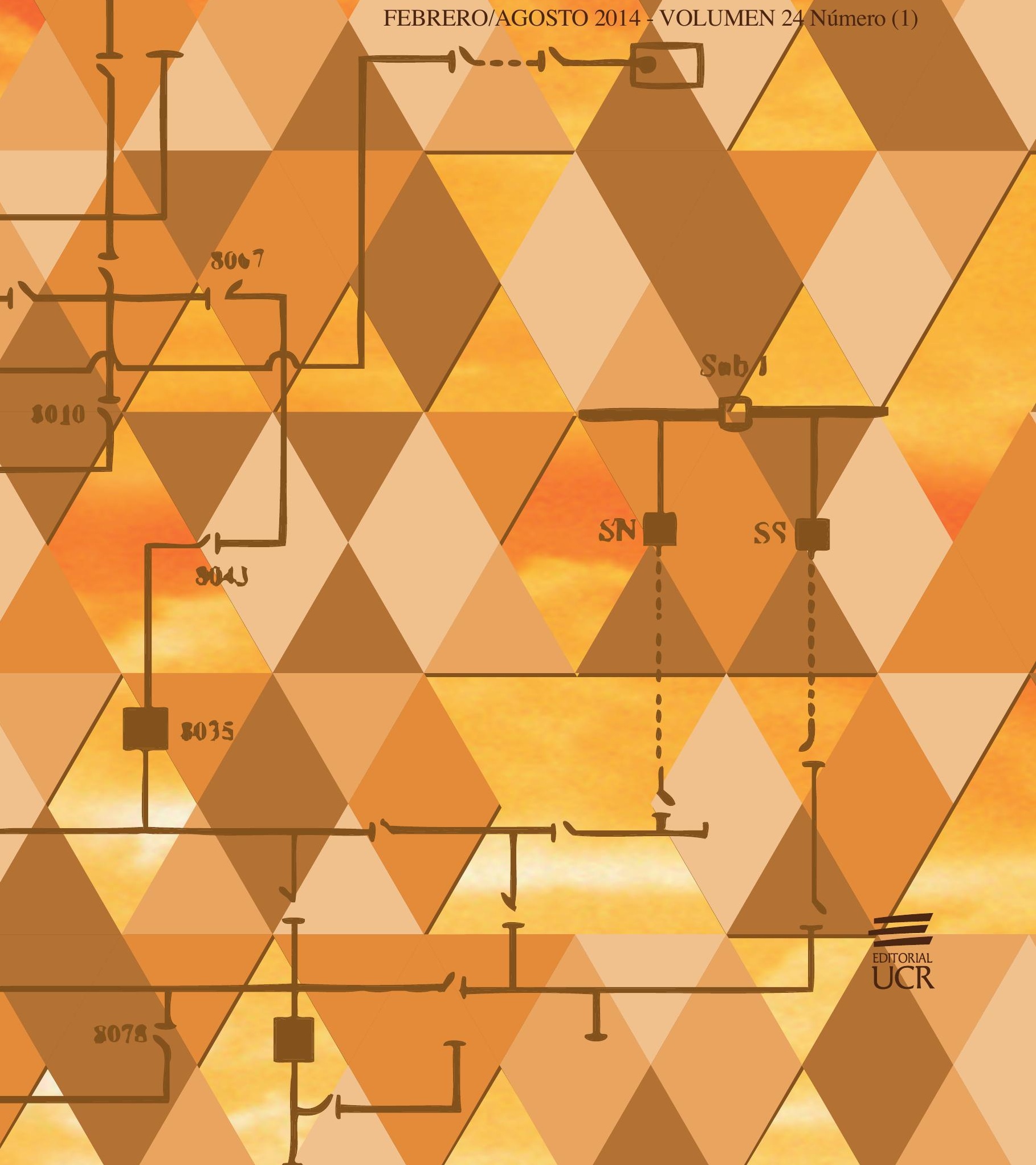




\title{
UN MODELO PARA ASEGURAR LAS MEDICIONES EN EL SISTEMA DE SALUD PÚBLICA DE COSTA RICA
}

\begin{abstract}
Resumen
Internacionalmente se realizan esfuerzos para asegurar las mediciones que se ejecutan en los distintos campos, de manera que sean correctas y trazables hasta referencias internacionales. Sin embargo, no hay evidencia de que el sistema de salud pública de Costa Rica logre articular esfuerzos para lograr una adecuada gestión de las mediciones realizadas por sus equipos médicos. Por lo cual, se propone un modelo que oriente y armonice los esfuerzos para asegurar las mediciones a nivel país y disminuir los riesgos asociados con las decisiones provenientes de las medidas realizadas con equipos médicos, más allá de lo estipulado en la ley para el Sistema Nacional para la Calidad.
\end{abstract}

Palabras Clave: Metrología Legal, equipo médico, metrología, riesgo, salud pública, Teoría de Redes.

\begin{abstract}
There are international efforts to develop the quality of the measurements in order to reduce risks upon decisions taken on different fields; on this way measurements systems reliability is improved. However, there is no evidence of articulated efforts to guaranty the measurements made by medical equipment in the Costa Rican public health national system. This article propone a model that guides and harmonizes the national efforts in order to diminish the associated risk on the decisions made with this equipment, beyond the provisions of the law for the National System for Quality.
\end{abstract}

Keywords: Legal Metrology, medical equipment, metrology, risk, public health, Nets Theory.

Recibido: 23 de junio de 2013 • Aprobado: 16 de enero 2014

\section{INTRODUCCIÓN}

Todos los días el personal de enfermería, médico y técnico, toma decisiones que pueden afectar la salud de las personas basados en las mediciones realizadas por diferentes instrumentos. En algunas ocasiones las utilizan para realizar un diagnóstico, en otras para ajustar el tratamiento adecuado, muchas veces sin preguntarse si la medición que indica el instrumento es correcta, pero ¿Hay alguien que se preocupe por esto? Jay L. Bucher (2008) en su artículo, "Asunto de vida o muerte", menciona que la magia se puede convertir en caos, cuando hace alusión a que una medición incorrecta en un equipo de alta tecnología para uso médico puede llevar a la muerte de los pacientes por más de un camino, desde la influencia directa hasta un diagnóstico errado que induzca un tratamiento inadecuado.

Los sistemas de mediciones y su entorno han sido ampliamente estudiados a través del tiempo, de hecho es una ciencia llamada metrología. Se entiende como metrología a la "Ciencia que tiene por objeto el estudio de los sistemas de pesas y medidas"(Real Academa Española, 2010) y por confirmación metrológica "Conjunto de operaciones requeridas para asegurarse que el equipo de medición es conforme a los requisitos correspondientes a su uso previsto." (Instituto de Normas Técnicas de Costa Rica (INTECO), 2003). 
En los últimos años la metrología a nivel internacional se ha preocupado por unificar los sistemas de mediciones y por encontrar estándares apropiados a los cuales trazar los patrones de referencia. (Jackson, 2006). De esta forma han nacido las diferentes asociaciones y organismos internacionales, regionales y nacionales que tratan el tema y procuran establecer una coordinación y ordenamiento a nivel global.

Este ordenamiento pretende facilitar la comunicación a nivel técnico de manera que los diferentes proveedores de equipos, servicios, materiales y usuarios a nivel mundial puedan llegar a una certeza de las diferentes mediciones, dimensiones, estándares.

En la industria médica la misma tendencia se mantiene, esta función se ha cargado en una rama de la ingeniería que se ha llamado de diferentes maneras, según la definición que sugiere García (2009), "la Electromedicina conocida como Biomédica o Ingeniería Clínica es la especialidad de las ciencias de la salud que estudia y analiza el cuidado de la Salud desde el punto de vista de la Tecnología Sanitaria.”. El doctor García aclara esta definición en una concepción más amplia: "consiste en la correcta planificación, aplicación y desarrollo de equipos y técnicas utilizadas en los exámenes y tratamientos médicos, así como el control de la calidad de los equipos empleados y la prevención de los riesgos asociados".(García, 2009).

Según la norma INTE- ISO 10012-2003 Sistemas de gestión de las mediciones Requisitos para los procesos de medición y los equipos de medición:

\footnotetext{
Un sistema eficaz de gestión de las mediciones asegura que el equipo y los procesos de medición son adecuados para su uso previsto y es importante para alcanzar los objetivos de la calidad del producto y gestionar el riesgo de obtener resultados de medición incorrectos" (INTECO, 2003).
}

Sin embargo, en muchos casos, esta responsabilidad de gestión de riesgos se circunscribe de una manera limitada, a la función única de calibración:

Calibración: operación que, en determinadas condiciones, en una primera etapa, establece una relación entre los valores de la magnitud de las incertidumbres de medición proporcionados por las normas de medición y las indicaciones correspondientes a las incertidumbres de medición asociadas y, en una segunda etapa, utiliza esta información para establecer una relación para obtener una resultado de la medición de una indicación. (Organización Internacional de Metrología Legal, 2013)

Los países dependen de diversas instituciones para lograr un sistema que asegure las mediciones de manera apropiada, para el bien de fabricantes, comerciantes y consumidores. La OEA en su libro "Metrología para no metrólogos" hace una explicación del tema y de las diferentes ramas de la metrología:

Las actividades relacionadas con la metrología dentro de un país son responsabilidad de una o varias instituciones autónomas o gubernamentales y, según sus funciones, se caracteriza como Metrología Científica, Legal ó Industrial dependiendo de su aplicación. (Marbán \& Pellecer, 2002)

La metrología legal es la "Práctica y proceso de aplicación de la estructura y el cumplimento legal y reglamentario a la metrología" (Organización Internacional de Metrología Legal, 2013). Debido a que se quiere revisar la participación de diferentes actores sociales en los procesos de gestión metrológica a nivel nacional, esta rama de la metrología es la que atiende mejor los conceptos discutidos. Esta ha sido regida internacionalmente por la Organización Internacional de Metrología Legal (OIML), que es una organización intergubernamental que desarrolla modelos regulación/legislación para sus miembros, según lo indica en su página web.

Esta misma organización aclara y establece la pertinencia de una "Infraestructura Metrológica Necesaria”, que permita una medición consistente por el bienestar de la industria y de los clientes, según ella misma define ${ }^{1}$ :

Ninguna cantidad puede ser medida consistentemente sin metrología y una infraestructura metrológica. La importancia de los resultados metrológicos esta en un continuo 
crecimiento debido al rápido desarrollo tecnológico y la información tecnológica emergente. Consumidores e industria deben tomar decisiones cada día basados en resultado de mediciones las cuales afectan su economía y su bienestar personal, también para juzgar las acciones y eficiencia de autoridades públicas, empresas y autoridades no gubernamentales. (OIML, 2013)

La Norma ISO 10012 Sistemas de gestión de las mediciones - Requisitos para los procesos de medición y los equipos de medición aborda el tema desde la perspectiva de procesos, da importancia a aspectos como compromiso de la gerencia y administración de recursos como los humanos, informáticos, materiales y proveedores externos; además de propiamente el tema de la confirmación metrológica y los procesos de medición. Esta norma tiene un capítulo completo dedicado al análisis y mejora del sistema de medición, sin embargo, no es de acatamiento obligatorio y está enfocada en la gestión metrológica de instituciones y empresas y no a modelos de nivel nacional.

Pese a esta limitación de alcance, esta norma es útil para determinar temas clave en gestión de sistemas de mediciones y cómo deben ser abordados por los actores sociales respectivos. Por ejemplo, los transversales a todos sus apartados como lo son: documentación, registros, demostración de capacidades y satisfacción de las necesidades de los clientes.

Relacionado con la satisfacción de las necesidades de los clientes, se encuentra el uso previsto del equipo médico y la confirmación metrológica. La confirmación metrológica, según la norma INTE - ISO 12000:2003 ${ }^{2}$ es un proceso que incluye los requerimientos de los clientes y su uso previsto, además de la calibración de este mismo equipo.

El riesgo de las mediciones incorrectas y sus efectos está relacionado básicamente con el uso previsto del equipo, tema en el que hace énfasis la norma, orientando su gestión al cliente. Sin embargo, no se queda solo ahí ya que hace diversas referencias a las consecuencias de estas medidas, así por ejemplo, entre los primeros requerimientos que la norma indica que se deben tener en cuenta son los riesgos del incumplimiento de los requisitos metrológicos.
La conexión entre la importancia de las mediciones y el riesgo asociado con las mediciones incorrectas parece evidente, más si se trata de equipo médico en cualquiera de sus usos. Lo que la Norma INTE-ISO 10012:2003 indica referente al uso previsto de los equipos y el riesgo asociado a las consecuencias de las mediciones incorrectas es totalmente válido y más que eso, apremiante cuando se trata de equipo médico.

En el ámbito costarricense, la aplicación de la confirmación metrológica y la mitigación de causas de riesgos en la biomedicina o cualquiera de sus ramas no están claramente estructuradas. Quién está llamado a normar y regular las actividades de prestación de servicios de atención directa a las personas es el Ministerio de Salud, esta institución ha legislado la tecnología referente a radiaciones ionizantes principalmente, y la importación de tecnologías médicas; sin embargo, esta legislación deja entrever vacíos en el tema metrológico y la mitigación de riesgos relacionada.

Adicionalmente, la Ley 8279 decreta el Sistema Nacional para la Calidad e instaura el Consejo Nacional para la Calidad (CONAC) con la participación de diferentes ministros y presidentes de cámaras de comerciantes y consumidores, entre otros, orientado a promocionar y difundir la calidad. Esta ley también crea, entre otros:

- El Laboratorio Costarricense de Metrología a quién le da la función de "Difundir y fundamentar la metrología nacional y promover el establecimiento de una estructura metrológica nacional" (Gobierno de Costa Rica, 2010).

- Ente Costarricense de Acreditación (ECA) encargado de realizar los procedimientos de acreditación en lo que respecta a laboratorios de ensayo y calibración.

- El Órgano de Reglamentación Técnica, encargado de la elaboración de reglamentos técnicos.

- El Ente Nacional de Normalización. Encargado de encausar y dirigir la elaboración de las normas convenientes para el desarrollo socioeconómico nacional, incluso la adopción de normas internacionales y la armonización en ámbitos supranacionales" (Gobierno de 
Costa Rica, 2010). Actualmente, según lo establece esta misma ley en el Transitorio III, se reconoce al Instituto de Normas Técnicas de Costa Rica (INTECO) como el Ente Nacional de Normalización.

Esta estructura es la base para la Metrología Legal en Costa Rica, debido a que integra las aristas técnica y legal para dar sustento a un sistema capaz de asegurar las mediciones y la posición para solicitar su cumplimiento obligatorio a través de la promulgación de leyes.

El principal referente a nivel nacional en prestación de servicios de atención directa a las personas es la Caja Costarricense de Seguro Social $^{3}$ (CCSS), no sólo por su inversión en equipo médico, sino también por la cantidad de dinero y esfuerzo que dedica para la capacitación de sus profesionales, investigación y mejora en sus tecnologías. Esta institución tiene metas claramente definidas en su documento:

\begin{abstract}
"Una CCSS Renovada hacia el 2025" así como políticas y estrategias que se proponen implementar, donde destacan el aumento en la cobertura a nivel nacional de diferentes servicios y tecnificación los servicios especializados. (Caja Costarricense de Seguro Social, 2007)
\end{abstract}

Estas metas implican un aumento indiscutible en la cantidad de equipos médicos que administrará esta institución, que ya al 2008 sumaban 204 mil millones de colones (Caja Costarricense de Seguro Social, 2009). Esta institución deja clara la necesidad de mejorar el nivel técnico de manejo de riesgo, dentro de sus políticas explicita la necesidad de mejorar el nivel técnico de sus funcionarios, y en el plan estratégico 2007 2010, una de sus acciones estratégicas enuncia el establecimiento de la Unidad de Gestión de Riesgos a nivel institucional. También se menciona la siguiente acción estratégica:

"Puesta en marcha del sistema institucional de evaluación de tecnologías sanitarias (Equipo médico, metodologías de diagnóstico, tratamientos médicos y quirúrgicos, medicamentos, etc) con el propósito de racionalizar su aplicación y uso, así como proteger la salud y seguridad de los pacientes". (Caja Costarricense de Seguro Social, 2007).

Aunque en las 141 actividades estratégicas enunciadas en su plan estratégico, la CCSS no hace referencia directa a metrología o temas relacionados, existe la clara conciencia de temas que son variables directamente relacionadas: riesgo, la seguridad de los pacientes, racionalización de uso de los equipos médicos y protección de la salud. Además, incluye otro tema relacionado y que no ha sido explicito en este documento: Salud e higiene ocupacional.

Estos esfuerzos aislados ponen en evidencia que temas como riesgo, seguridad de pacientes y del personal, se están manejando en diversos actores sociales del sistema de salud pública costarricense, sin embargo, aun existen dudas razonables respecto a la armonía y coherencia de estos esfuerzos y la inclusión del aseguramiento de las mediciones como una variables crítica para lograrlos.

En resumen, la normativa y referencias internacionales refuerzan la necesidad de los países de contar con una organización suficiente para asegurar que las mediciones que se realicen sean adecuadas y reduzcan el riesgo asociado a las decisiones que se toman con ellas. Las decisiones que se toman con las mediciones realizadas con equipos médicos pueden decidir entre la vida o muerte de los pacientes a diario, y no parece ser que en Costa Rica el sistema de salud pública, esté articulado con una estructura que asegure las mediciones de estos equipos. La intención de este artículo es proponer un modelo que oriente los esfuerzos para asegurar que las mediciones que se realizan actualmente en este sistema de salud pública, y que este orientado a disminuir el riesgo en la prestación de servicios de atención directa a las personas.

\section{METODOLOGÍA}

Para la propuesta del modelo se identificaron las variables básicas que deben interactuar y los actores sociales que las proveen. Para esto se realizó un levantamiento de información que tuvo dos etapas debido a la dificultad para recabarla. 
Primero, se aplicó una encuesta estructurada y auto aplicada, dirigida a responsables del mantenimiento de equipo, los responsables del proceso de compra en los centros de prestación de servicios médicos y las empresas proveedoras de equipo médico.

La encuesta auto aplicada fue respondida por 21 personas de las cuales 16 pertenecen a la Caja Costarricense del Seguro Social y 5 a empresas que venden equipo y dan servicio de mantenimiento. Se utilizó para recabar información de los niveles operativos de mantenimiento de equipos, venta de equipos, prestadores de servicios.

Luego se aplicaron entrevistas semiestructuradas a otros actores sociales, como por ejemplo, tomadores de decisiones a nivel gerencial en los prestadores de servicios y el regulador nacional (Ministerio de Salud). También, se realizaron entrevistas semiestructuradas a los actores de la estructura metrológica nacional según el Sistema Nacional para la Calidad y referencias que ellos mismos suministraron como socios en esta labor, dentro de los organismos que respondieron a esta herramienta están: Laboratorio Costarricense de Metrología, laboratorios de referencia nacional en diferentes mediciones y otros laboratorios e instituciones de importancia nacional. Se determinó la saturación de la consulta, con un total de 37 personas entrevistadas.

La elaboración de estas herramientas estuvo basada en las normas técnicas y legislación, conocimiento sobre la estructura de las organización y teoría sobre calibración y metrología.

La identificación de las variables, los actores y las interrelaciones permitió establecer un modelo que dirija la administración del riesgo inherente a los equipos médicos y los componentes de medición que tienen asociados, siempre relacionado con la toma de decisiones médicas y las condiciones de seguridad de los pacientes y los usuarios de los equipos. Además se incluyó el estudio de redes para comprender, valorar y describir los sistemas que debían proponerse en términos de la confirmación metrológica, gestión de calidad y riesgo.

\section{MODELO DE ASEGURAMIENTO METROLÓGICO NACIONAL PARA EQUIPO MÉDICO}

\subsection{Análisis de los resultados obtenidos}

Las entrevistas evidenciaron que los participantes en el proceso identifican la necesidad fortalecer el marco normativo, se reconoce como una necesidad la existencia de más políticas y legislación referentes a metrología. Los únicos procedimientos identificados son de calibración, específicamente orientados a balanzas y esfigmomanómetros, este último relacionado con un reglamento nacional, único reconocido por las autoridades como oficializado a la hora de realizar la investigación.

Las personas y organizaciones contactatadas, incluyendo al Sistema Nacional para la Calidad, identificaron gran cantidad de actores sociales que deberían intervenir y de variables que deben activar el sistema en el sector de salud pública costarricense, sin embargo, conforme pasaron las entrevistas las opciones propuestas se volvieron repetitivas y se saturó la consulta.

Los actores propuestos se agruparon para simplificar su análisis, como se muestra a continuación:

- Estructura metrológica: son todos aquellos actores que están explícitamente relacionadas con la estructura metrológica nombrada por la OIML. En el caso de Costa Rica se refiere a entes explícitamente instaurados por la Ley 8279 Sistema Nacional para la Calidad, como el Laboratorio Costarricense de Metrología (LACOMET), el Ente Nacional de Normalización, representado por el Instituto de Normas Técnicas de Costa Rica (INTECO), y el Ente Costarricense de Acreditación (ECA).

- Prestadores de servicios médicos: personas, organizaciones o instituciones encargadas de brindar los servicios de atención médica a las personas. El principal prestador de servicios de atención médica a las personas en Costa 
Rica es la Caja Costarricense de Seguro Social (CCSS).

- Regulador: Institución a nivel nacional encargada de dictar políticas y legislación en el tema de su competencia. El regulador en temas de salud pública en Costa Rica es el Ministerio de Salud.

- Formadores: Institución encargada de formar a los profesionales en el tema de estudio. Principalmente universidades y organizaciones de educación superior.

- Otros actores sociales: Demás personas, grupos organizados, empresas, cualquier integrante de la sociedad, que pueda o deba hacer aportes, contrapeso técnico o científico, en favor del crecimiento del tema en estudio. Se han mencionado colegios profesionales, empresas privadas nacionales o internacionales, principalmente las dedicadas a manufactura de equipo médico, profesionales en el campo de la metrología, laboratorios de calibración, entre otros.

Estos actores son los encargados de poner en juego las variables que conforman el modelo. Son las líneas que dan cohesión a la red, dictan las interacciones que deben unir a los actores sociales que forman la estructura del sistema. Las variables identificadas se resumen a continuación:

- Capacitación: Conocida en este agrupamiento como todas aquellas actividades orientadas a crear conciencia, formar, transferir conocimiento y diseminar el mismo.

- Coordinación: Las acciones tendientes a crear sinergia entre distintos actores relacionados con el tema. Esto incluye coordinación de la participación social inteligente, trabajo en equipos interdisciplinarios para apoyar la toma de decisiones, acreditación y participación de actores sociales, alianzas internacionales para cooperación.

- Estructura metrológica: Temas que están explícitamente relacionadas con la estructura metrológica nombrada por la OIML, que incluye regulación, aplicación de normativa internacionalmente aceptada, calibraciones, existencia de patrones de referencia, entre otros. Estos deberían permitir, específicamente para el caso de los equipos de medición utilizados para los sistemas de salud pública: control metrológico legal, control legal de instrumentos de medición, supervisión metrológica y evaluaciones de conformidad, entre otros; de acuerdo a los conceptos establecidos por la Organización Internacional de Metrología legal y especificados en el Vocabulario internacional de términos en metrología legal (VIML) (OIML, 2013)

- Tecnología: Los métodos, herramientas, instrumentos, equipos que impliquen adelantos en conocimiento. Incluye equipamiento e infraestructura para realizar calibraciones, procedimientos para control del mantenimiento y procedimientos para el control de la calidad.

Se siguió la lógica de Teoría de Redes para articular el modelo, los actores son los nodos de la red, mientras que las variables los enlaces.

La tercera característica requerida en esta teoría y que hace falta para completar el modelo es la dirección de estos enlaces. Para completar esta propuesta se hace explícita la interacción entre los actores y las variables asociadas, esta interacción se basa en los criterios expresados por los actores sociales entrevistados, la legislación existente y la abstracción del autor. Estas relaciones se pueden apreciar en elTabla resumen, denominado matriz desde - hacia (Tabla 1).

Del Tabla 1 se desprenden varios aspectos importantes:

1. Los prestadores de servicios de salud son los grandes receptores del modelo, en su columna se puede apreciar cómo todos los demás actores deberían entregar estructura metrológica, tecnología, coordinación y formación, es decir, este actor debería recibir todas las variables, desde los otros actores, y los otros actores deberían desarrollar sus actividades en función de este gran cliente.

2. Los formadores y la estructura metrológica deberían ser actores protagonistas en este 
Tabla 1. Matriz desde-hacia que muestra la realción entre nodos y variables del sistema.

\begin{tabular}{|c|c|c|c|c|c|c|}
\hline & & & & Hacia & & \\
\hline & & $\begin{array}{l}\text { Prestadores de } \\
\text { servicios }\end{array}$ & $\begin{array}{l}\text { Estructura } \\
\text { metrológica }\end{array}$ & Reguladores & $\begin{array}{c}\text { Formadores } \\
\text { (Universidades) }\end{array}$ & Otros actores \\
\hline \multirow{5}{*}{ Desde } & $\begin{array}{l}\text { Prestadores de } \\
\text { servicios }\end{array}$ & -- & -- & -- & -- & -- \\
\hline & $\begin{array}{l}\text { Estructura } \\
\text { metrológica }\end{array}$ & $\begin{array}{c}\text { Estructura/ } \\
\text { Tecnología/ } \\
\text { Coordinación }\end{array}$ & -- & Coordinación & Coordinación & Coordinación \\
\hline & Reguladores & $\begin{array}{l}\text { Estructura } \\
\text { metrológica }\end{array}$ & $\begin{array}{l}\text { Estructura } \\
\text { metrológica }\end{array}$ & -- & -- & $\begin{array}{l}\text { Estructura } \\
\text { metrológica }\end{array}$ \\
\hline & $\begin{array}{c}\text { Formadores } \\
\text { (Universidades) }\end{array}$ & Formación & Formación & Formación & -- & Formación \\
\hline & Otros actores & Tecnología & Tecnología & --- & -- & -- \\
\hline
\end{tabular}

modelo, que deberían brindar, formación el primero y coordinación, estructura metrológica y tecnología el segundo. Es muy importante señalar cómo la estructura metrológica debería coordinar esfuerzos con los capacitadores, para lograr el fortalecimiento de las capacidades y competencias en los profesionales, de acuerdo a lo requerido por la sociedad en este tema específico.

3. Los grandes responsables de que la estructura metrológica se dé son los reguladores. Esto debido a la voluntad política y la facilidad para que los entes técnicos dispongan de los recursos necesarios, es de los reguladores. De estos depende la legislación que refuerce y obligue la disminución de los riesgos.

4. Existe en los otros actores, tecnología disponible que puede ayudar en gran medida en la ejecución y crecimiento de sistemas que ayuden a disminuir el riesgo asociado a las mediciones.

\subsection{Implicaciones prácticas}

\subsubsection{Etapa 1. Identificación de necesidades de los clientes y de requisitos de calidad.}

La concepción práctica debe iniciar con el cliente y usuario del servicio. De las variables y actores identificados en el apartado anterior se deben definir requisitos de calidad, requerimientos de uso y riesgos asociados con las mediciones realizadas con el equipo médico.

En esta primer parte los actores principales son los prestadores de servicios de salud, los actores sociales inteligentes y los entes reguladores. Estos se deben integrar para identificar la necesidad de medición asociada con el equipo y determinar la tecnología apropiada para su satisfacción.

En esta etapa se requiere mucho trabajo en equipos multidisciplinarios y la participación de las autoridades reguladoras que definan políticas tecnológicas, y de ser posible integren 


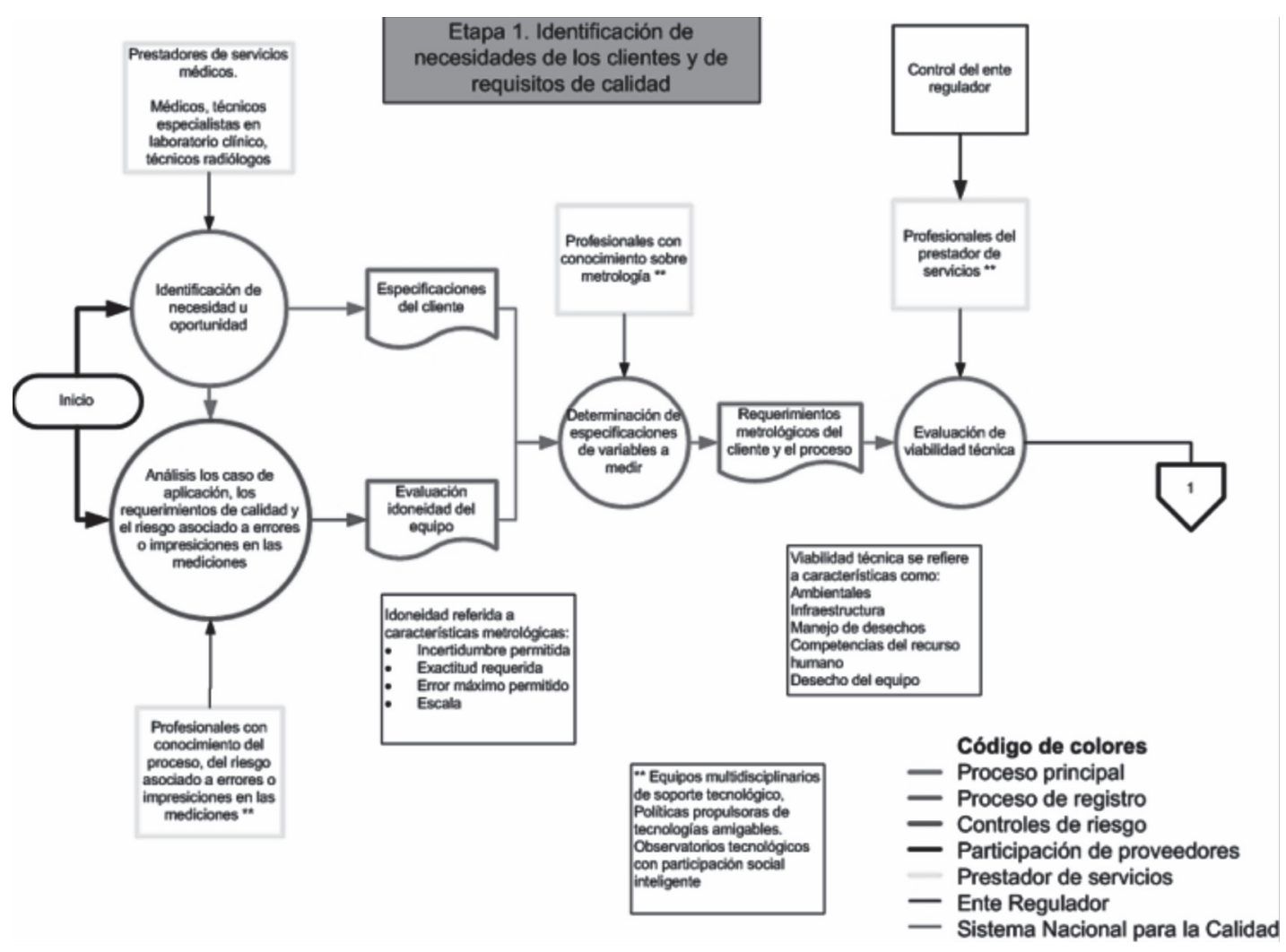

Figura 1. Modelo de aseguramiento de las mediciones. Etapa 1, indentificación de las necesidades de los clientes y requisitos de calidad.

los diferentes actores sociales como empresas privadas, universidades y colegios profesionales, para que se identifique, de manera pro activa, la tecnología que más le conviene al país en cada uno de los temas de interés, y la investigación y capacitación de los demás actores sociales, de acuerdo a la conveniencia específica. La Figura 1 muestra las acciones, algunos actores e interacciones relacionadas con esta parte.

En esta parte del sistema se deben identificar de manera sistémica los riesgos y plasmar en los requerimientos metrológicos, se debería dejar claro cuáles son las variables suficientes y adecuadas para mitigar el riesgo, de manera que en el futuro las capacitaciones, el uso y las confirmaciones metrológicas del equipo sean incorporados de manera adecuada.

\subsubsection{Etapa 2. Selección, compra e instalación de equipos}

Los actores sociales representados por fabricantes, vendedores e instaladores deberían adquirir un papel protagónico en esta etapa. Los organismos reguladores también lo hacen a nivel del control, ejercido desde dos puntos de vista: 


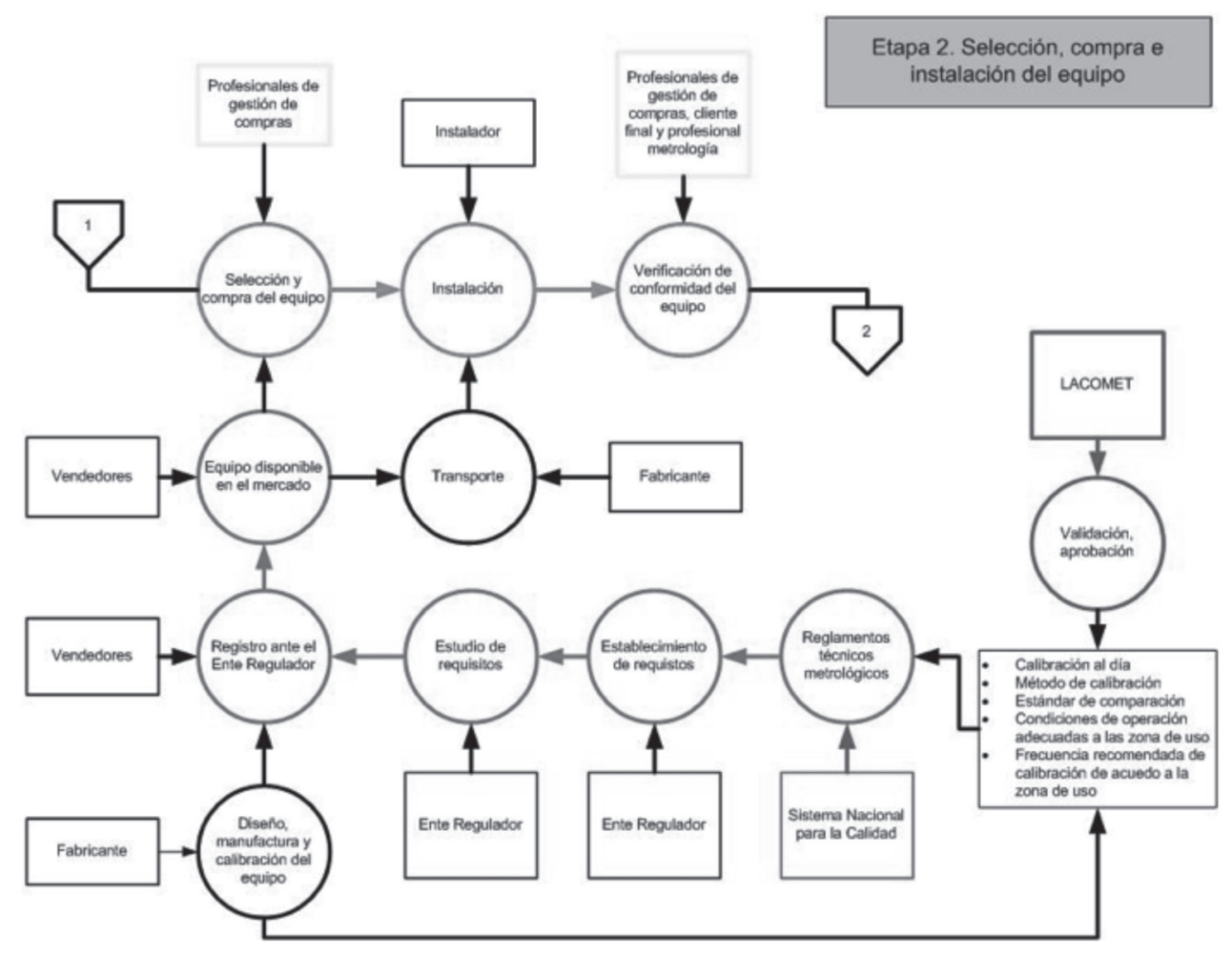

Figura 2. Modelo de aseguramiento de las mediciones. Etapa 2, selección compra e instalación de equipos.

i. Registro de equipo y definición de requerimientos de entrada al mercado.

ii. Control de cumplimiento de condiciones de operación para la instalación del equipo.

El personal de mantenimiento del prestador de servicios debería estar capacitado en las tecnologías y los equipos de manera tal, que pueda ser una contraparte capaz de cuestionar las diferentes situaciones relacionadas con la selección, compra y puesta en marcha del equipo. Las autoridades reguladoras del país, de igual manera capacitadas para cuestionar y evaluar la conveniencia de la tecnología y lo adecuado de las condiciones de uso planteadas. La metrología debe ser parte del control de calidad de la función de mantenimiento, en este caso, de la instalación y puesta en marcha.

En este punto se pone en evidencia las dos necesidades básicas del sistema:

i. La necesidad de conciencia y conocimiento técnico relacionado a aspectos de metrología en todos los nodos del sistema.

ii. La necesidad de concientizar, fomentar y coordinar los esfuerzos en el desarrollo de capacidades, es decir, articular el esfuerzo de los capacitadores con las necesidades del mercado, para que se desarrollen las competencias que la sociedad requiere.

El diagrama que explica esta parte del modelo se presenta en la Figura 2. 


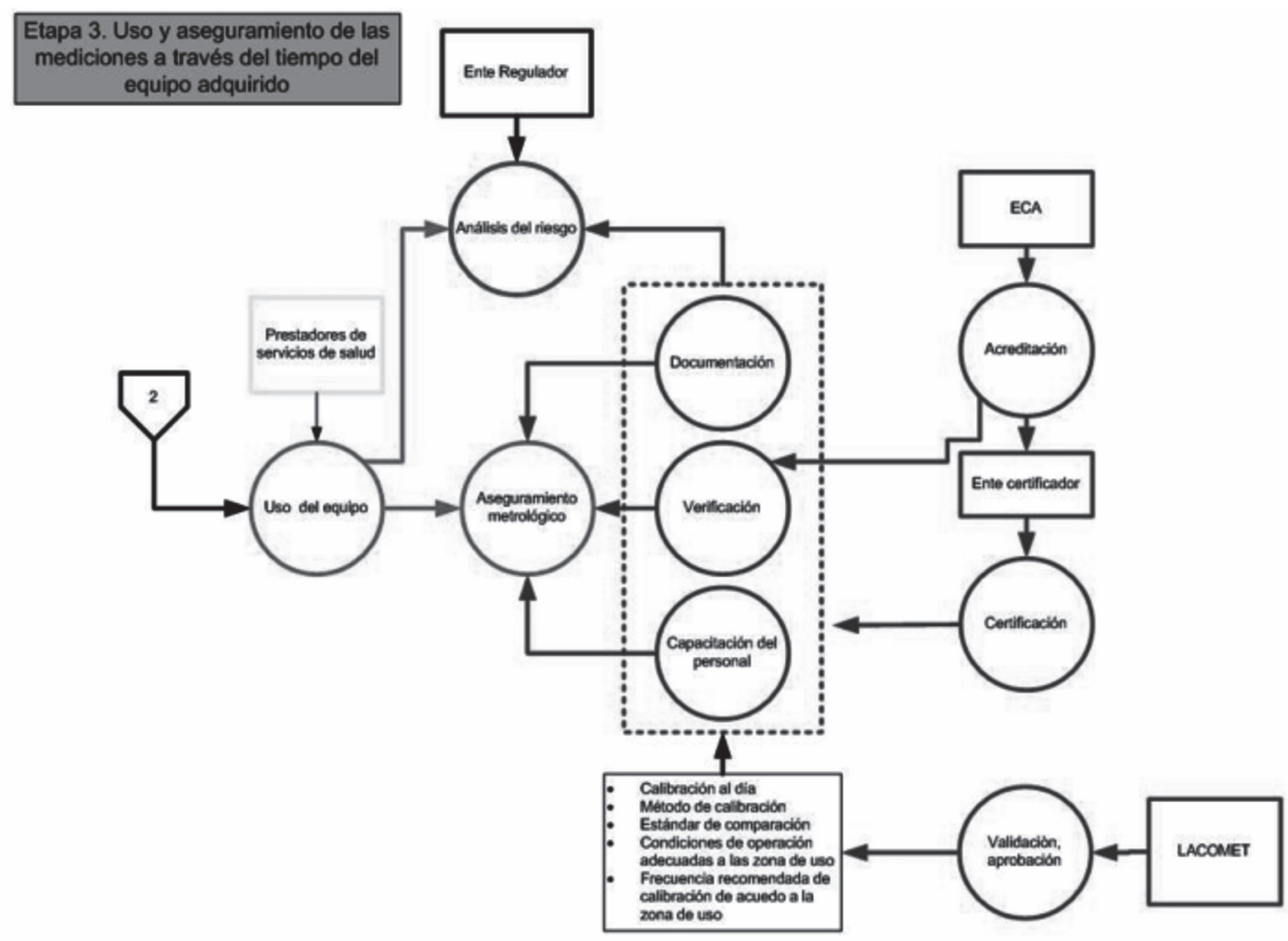

Figura 3. Modelo de aseguramiento de las mediciones. Etapa 3, uso y aseguramiento metrológico a través del tiempo.

\subsubsection{Fase 3. Uso y aseguramiento metrológico a través del tiempo.}

La tercer parte y final de esta propuesta implica el estudio del uso y evaluación metrológica del equipo.Cuando el equipo entra en funcionamiento, el estado del arte en metrología nos indica que existe normativa internacional y mucho conocimiento en este tema, de manera genérica y específica.

En Costa Rica propiamente, se han adoptado algunas de estas normas, sin embargo su uso es de carácter voluntario. Esta limitante se une a la falta de conocimiento de muchos de los sectores de nuestra sociedad,de recursos y conocimiento en los entes llamados a regular y poner en práctica las medidas de mitigación, de manera que se generan vacíos en la aplicación de accionesque disminuyan los riesgos relacionados a posibles mediciones incorrectas.

Una vez más los actores principales de esta etapa son los prestadores de servicios de salud, pero se propone una intervención más protagónica de la estructura metrológica del país, representada en este caso por el Ente Costarricense de Acreditación (ECA), el Laboratorio Costarricense de Metrología (LACOMET) y el Instituto de Normas Técnicas de Costa Rica (INTECO). En este punto es necesario resaltar la participación de los entes reguladores en el análisis de riegos del sistema. 


\section{CONCLUSIONES}

Los esfuerzos en metrología realizados en la actualidad para lograr el aseguramiento de las mediciones en los equipos de uso médico del sistema de salud pública de Costa Rica son insuficientes para lograr la adecuada administración de los riesgos inherentes. La falta de conciencia y capacidad técnica en el tema por muchas de las personas que participan en la cadena de regulación y toma de decisiones sobre la adquisición, uso y mantenimiento de equipo médico, adicionado a la falta de asignación de recursos por las autoridades, hacen que actualmente exista una deficiencia en la gestión de los riesgos relacionados a las mediciones hechas por equipos médicos en el país.

Es imperante que los entes estatales, ya sea prestadores de servicios o los entes reguladores y la estructura metrológica, articulen a los actores sociales inteligentes para poder potenciar en un plazo no lejano los esfuerzos en metrología. El crecer en la conciencia, conocimiento técnico y adopción de medidas que refuercen el control metrológico requiere de una participación activa y decidida de actores que no necesariamente son evidentes en este proceso, como por ejemplo las universidades, proveedores de equipo, industria tecnológica y los colegios profesionales y reforzado con regulación y recursos para acuerpar los pocos reglamentos y leyes que actualmente se relacionan con estos temas.

Es indispensable fortalecer la normativa legal existente en el país para establecer requisitos vinculantes en el tema y establecer parámetros que permitan actividades propias de la metrología legal como: el control metrológico legal, control legal de instrumentos de medición y supervisión metrológica.

El enfoque de análisis exploratorio que caracteriza este estudio, lleva al planteamiento desde una perspectiva macro, de una red de soporte que funcione como un sistema. Sin embargo, aun existen muchas oportunidades de investigación, por ejemplo las dirigidas a analizar las condiciones y posibilidades existentes en: i. La interacción de los entes reguladores, las universidades y los colegios profesionales en el desarrollo de capacidades de los profesionales vinculados con la toma de decisiones que involucran mediciones realizadas con los equipos médicos.

ii. La interacción de una sociedad en desarrollo, cómo la costarricense, con empresas transnacionales y la oportunidad que esto representa de tener acceso a diferentes tecnologías. Cómo estas pueden impactar el desarrollo de capacidades internas, legislación, investigación, conciencia de la sociedad. Adicionalmente a otros vínculos con autoridades a nivel internacional, que le permitan al país, potenciar su capacidad como sociedad, específicamente en los temas de metrología en el sector salud.

iii. La necesidad de potenciar la conciencia y competencias desarrolladas en la educación básica del país, regida por el Ministerio de Educación Pública, en cuanto a la metrología y su importancia.

iv. El vínculo entre los diferentes entes reguladores, las universidades y colegios profesionales tendiente a potenciar la interacción entre el mantenimiento de los equipos médicos y la evaluación de sus características metrológicas.

v. Las oportunidades de generar sinergia entre los entes establecidos por el Sistema Nacional para la Calidad, como alinear los esfuerzos y objetivos y armonizar las acciones relacionadas con sus socios naturales, con el fin de mejorar de la calidad en el país, específicamente en lo referente a los temas de metrología en el sector salud.

\section{NOTAS}

1. Traducción del autor.

2. Versión de la norma ISO 100012, actualmente vigente en Costa Rica, adaptada y aceptada por el Instituto Costarricense de Normas Técnicas (INTECO). 
3. El modelo de Salud Pública de Costa Rica separa al organismo rector de la salud pública (Ministerio de Salud) de los prestadores de servicios médicos hospitalarios (En este caso la Caja Costarricense de Seguro Social).

\section{BIBLIOGRAFÍA}

Ávalos, A. (15 de Julio de 2006). Demanda por daño moral por accidente con bomba de cobalto. La Nación.

Caja Costarricense de Seguro Social. (2007 15-Noviembre).Plan Estratégico Institucional 2007 - 2010. San José, San José, Costa Rica.

Caja Costarricense de Seguro Social. (2009 17-abril). Seguro de Enfermedad $y$ Maternidad Estados Financieros Auditados. San José, San José, Costa Rica.

Caja Costarricense de Seguro Social. (2007). Una CCSS Renovada hacia el siglo 2025. San José, San José, Costa Rica. Recuperado de: http://www.ccss.sa.cr/

García, J. (2009). Formación Básica en Ingeniería Biomédica. Curso: Formación Básica en Ingeniería Biomédica. San José: Universidad de Costa Rica.

Gobierno de Costa Rica. (17 de 03 de 2010). Ley 8279 Sistema Nacional para la Calidad. San José, Costa Rica: Gobierno de Costa Rica.

Instituto de Normas Técnicas de Costa Rica (INTECO). (2003). Inte-ISO 10002-2003. Sistemas de gestión de las mediciones. Requisitos para los prcesos de medición y los equipos de medición. San José, Costa Rica: INTECO.
Jackson, C. (2006). Annual Meeting - Joint Committee for traceability in Laboratory Medicine. Quality Assurance, 642-643.

Marbán,R., \& Pellecer, J.(2002). Metrología para no metrólogos (2da edición ed.). Guatemala: Organización de Estados Americantos.

Organización Internacional de Metrología Legal. (s.f.). Recuperado el 28 de 7 de 2011, de www.metrologyinfo.org/oiml-1.html

Organización Internacional de Metrología Legal. (2004). Elements for a Law on Metrology. Recuperado el 27 de Julio de 2011, de http:/ www.oiml.org

Organización Internacional de Metrología Legal. (2013). Vocabularion internacional de términos en metrología legal (VIML). (O. i. legal, Ed.)

Real Academa Española. (2010,11-Enero). Diccionario de la Real Academia Española. Retrieved 2010, 11-Enero from Real Academia Española Web site: http://www. rae.es/rae.html

Webster, J. (2006). Metrología Olográfica, algunos ejemplos de reportes de imágenes en medicina y pruebas no destructivas. The imagine Sciencs Journal, 54, 80-85.

\section{SOBRE EL AUTOR}

Ronny Pacheco Segura, Universidad de Costa Rica, docente en la Escuela de Ingeniería Industrial. Máster en Ingeniería Industrial, Correo electrónico: ronny.pacheco@ucr.ac.cr, teléfono: 8827-97-42. 